\title{
Comment on the European position paper on diagnostic tools in rhinology - computational fluid dynamics*
}

\author{
Eugene Wong' ${ }^{1}$ Kiao Inthavong², Narinder Singh' \\ Department of Otolaryngology, Head and Neck Surgery, Westmead Hospital, Sydney Medical School, Australia \\ 2School of Engineering, RMIT University, Melbourne, Australia
}

\author{
Rhinology 57: 6, 477 - 478, 2019 \\ https://doi.org/10.4193/Rhin19.269 \\ *Received for publication: \\ August 9, 2019 \\ Accepted: September 1, 2019
}

\section{Dear Editor,}

We read with interest the excellent paper authored by Rimmer et al. (1) entitled The European Position Paper on Diagnostic Tools in Rhinology. The authors are to be commended for their comprehensive, up-to-date and thorough summary of available tests in the assessment of rhinologic function. The paper describes traditional tools used in nasal airway obstruction (NAO); including subjective patient reported outcome measure questionnaires (PROMs) such as the NOSE scale; tests that are subjective to the clinician, such as clinical examination, nasendoscopy and imaging; and objective tests such peak nasal inspiratory flow (PNIF), rhinomanometry (RM) and acoustic rhinometry (AR). Unfortunately, each of these readily available, traditional tools fail to meet several accepted criteria of an ideal diagnostic test (Table 1), as outlined ${ }^{(2,3)}$. In our opinion, these limitations restrict the capacity of Rhinology to develop as a discipline founded on sound evidence-based science.

Computational Fluid Dynamics (CFD) is a modern scientific tool that has been gaining popularity in the assessment of rhinologic disease and in particular for NAO ${ }^{(4)}$. CFD utilises the same technology used to assess the aerodynamic characteristics of racing cars and aircraft ${ }^{(5)}$. Using the paranasal sinuses as a fixed model, simulation of nasal airflow, sinus ventilation and behaviour of topical drug delivery can be performed. In addition to assessing NAO in an accurate, reproducible, quantifiable, validated and clinically relevant manner that precisely identifies the site(s) of obstruction, CFD can also be used to accurately assess pressure, velocity, wall shear stress and temperature changes throughout the sinonasal cavity ${ }^{(6)}$.

Therefore, CFD does not carry many of the limitations of the traditional rhinologic assessment tools, and carries significant potential in the rhinologist's search for a new era of data-driven scientific discovery. Over the last 15 years, there has been exponential increase in sinonasal CFD publications with over 80 papers annually in peer-reviewed journals. The Society for CFD of the Nose and Airway (SCONA, www.scona.org) has held two world congresses, in association with ERS London, 2018 and Rhinoworld, Chicago, 2019. Clinical use of CFD has traditionally required collaboration between otolaryngologists and fluid dynamics engineers. However, new tools designed for everyday clinical use by otolaryngologists have been described and are currently being developed by several groups internationally ${ }^{(7,8)}$.

We would respectfully suggest that the excellent position paper on Diagnostic Tools in Rhinology would benefit by including a description of CFD in any future updates or addenda.

\section{Acknowledgements}

None to declare

\section{Authorship contribution}

All authors (EW, KI, NS) contributed to conception of the paper and manuscript preparation.

\section{Conflict of interest}

No conflicts of interest exists.

Table 1. Author's suggestions for characteristics of an ideal diagnostic or screening test in rhinology.

\section{Objective}

Accurate (Sensitive and Specific)

Low-risk

Acceptable

Provides diagnostic insight

Captures the effect of interventions

Readily available

Economically feasible

Reliable/Valid

Consistent/ Reproducible

Discriminates well

Permits useful comparisons 


\section{References}

1. Rimmer J, Hellings $P$, Lund V, Alobid I, Beale T, Dassi C et al. European position paper on diagnostic tools in rhinology. Rhinology. 2019 Supp 28:1-41

2. Maxim LD, Niebo R, Utell MJ. Screening tests: a review with examples. Inha Toxicol.2014 Nov; 26(13):811-828.

3. Bossuyt PM, Reitsma JB, Linnet K, Moons KG. Beyond diagnostic accuracy: the clinical utility of diagnostic tests. Clin Chem. 2012 Dec; 58(12):1636-1643.

4. Leong SC, Chen XB, Lee HP, Wang DY. A review of the implications of computational fluid dynamics studies on nasal airflow and physiology. Rhinology.2010 Jun; 48(2):139145.
5. Agarwal RK. Computational fluid dynamics of whole-body aircraft. Annual Review of fluid Mechanics. 2003 Nov; 31(1):125-169.

6. Wang DY, Lee HP, Gordon BR. Impacts of fluid dynamics simulation in study of nasal airflow physiology and pathophysiology in realistic human three-dimensional nose models. Clin Exp Otorhinolaryngol.2012 Dec; 5(4):181-187.

7. Vanhille DL, Garcia GJM, Asan O, Borojeni AAT, Frank-Ito DO, Kimbell JS et al. Virtual surgery for the nasal airway: a preliminary report on decision support and technology. JAMA Facial Plast Surg.2018 Jan 1; 20(1): 63-69.

8. Burgos MA, Sanmiguel-Rojas E, Singh N Esteban-Ortega F. Digbody: a new 3D mod elling tool for nasal virtual surgery. Comput Biol Med.2018 Jul 1; 98: 118-125.

Dr Eugene Wong

Department of Otolaryngology

Head and Neck Surgery

Westmead Hospital

Sydney Medical School

Australia

E-mail: eugene.hl.wong@gmail.com 